\title{
Impact of electron-scale turbulence and multi-scale interactions in the JET tokamak
}

\author{
N. Bonanomi ${ }^{1,2,4}$, P. Mantica ${ }^{2}$, J. Citrin ${ }^{3}$, T. Goerler ${ }^{4}$, B. Teaca ${ }^{5}$ and JET contributors* \\ EUROfusion Consortium, JET, Culham Science Centre, Abingdon, OX14 3DB, UK \\ 1) Università di Milano-Bicocca, Milano, Italy \\ 2)CNR - Istituto di Fisica del Plasma "P. Caldirola", Milano, Italy \\ 3)DIFFER - Dutch Institute for Fundamental Energy Research, $5600 \mathrm{HH}$, Eindhoven, The Netherlands \\ 4)Max Planck Institute for Plasma Physics, Boltzmannstr. 2, 85748 Garching, Germany \\ 5)Applied Mathematics Research Centre, Coventry University, Coventry CV1 5FB, United Kingdom \\ * See the author list of "X. Litaudon et al., Nucl. Fusion 57, 102001, (2017)"
}

\begin{abstract}
Experimental observations in JET tokamak plasmas and gyrokinetic simulations point to an important role, for electron heat transport, of electron-scale instabilities and of their interaction with ion-scale instabilities. Since these effects are maximized for strong electron heating and ion-scale modes close to marginal stability, these findings are of high relevance for ITER plasmas, featuring both conditions. Gyrokinetic and quasi-linear transport models accounting for multi-scale effects are assessed against JET experimental results.
\end{abstract}

Introduction: Understanding turbulent electron heat transport is of fundamental importance for future magnetic fusion reactors such as ITER. Both the main heating systems and, in a Deuterium-Tritium plasma, the fusion burn $\alpha$-particle heating will deliver power mainly to the electron channel, whilst ions will be mainly heated by electrons via collisional coupling. Therefore, the core temperature and fusion performance will strongly depend on electron heat transport, which is dominated by turbulent processes. Historically, ion-scale micro-instabilities, i.e. ITG (Ion Temperature Gradient) modes [1] and TEM (Trapped Electron Modes) [2], have been deemed to cause most of the electron heat flux, $q_{e}[3,4,5]$, with electron-scale ETG (Electron Temperature Gradient) modes [6] playing a minor role due to their small wavelength. However, a quantitative comparison with non-linear gyrokinetic (GK) simulations of the experimental $q_{e}$ and its slope vs $R / L_{T e}=R|\nabla T e| / T e$ ( $R$ being the plasma major radius and $T_{e}$ the electron temperature) - which determines the stiffness of the $T_{e}$ profiles against a flux increase - was attempted only very recently. This pointed out that, in some cases, ion-scale low-k modes are not able to account for the measured electron heat transport [7, 8, 9]. Both theoretical $[6,8,10,11,12]$ and experimental [13, 14, 15] studies, including studies in spherical tokamaks $[16,17,18,19]$, extending to the high-k range have then shown that, in some experimental conditions, ETGs can carry a significant fraction of the turbulent heat flux and electron and ion scales can have strong interactions. The importance of sub-ion instabilities and of the interactions between different scales has also been investigated and observed in other magnetized plasma phenomena, such as in magnetic reconnection [20, 21] and solar wind [22]. In fusion plasmas, it was found in computationally demanding multi-scale GK simulations, including a simulation of a DIII-D ITER baseline discharge with dominant electron heating [23], that a large impact of ETG modes takes place when ion-scale instabilities are close to marginal stability and high electron heating is present $[8,11]$. This will be the case in the ITER baseline scenario, motivating the present study on JET, the largest existing tokamak. We focus on JET L-mode plasmas with high electron heating, on which we performed dedicated transport experiments, comparing results with GK simulations. The target of the work is to quantify the 


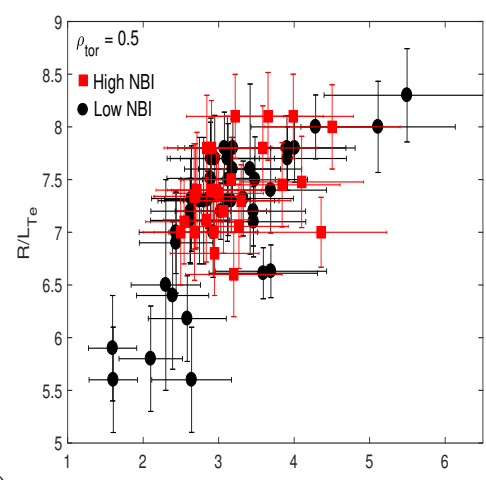

a)

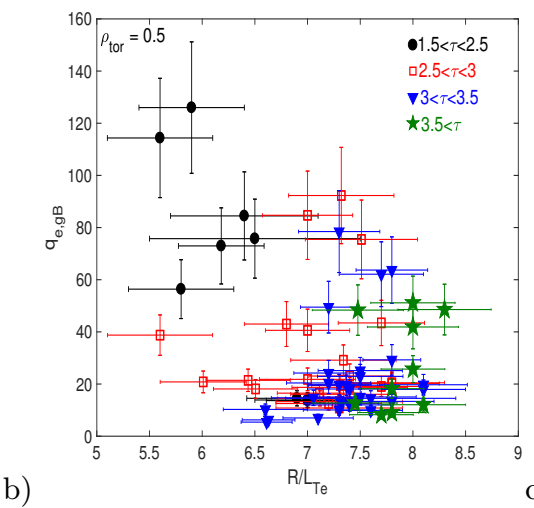

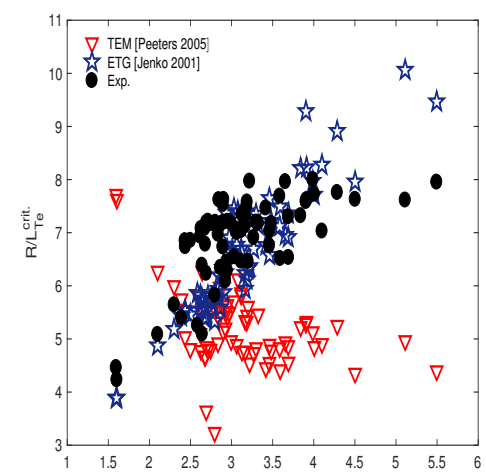

Figure 1) a) $R / L_{T e}(\tau)$ with high (red) and low (black) NBI at $\rho_{\text {tor }}=0.5$. b) $q_{e, g B}\left(R / L_{T e}\right)$ at $\rho_{\text {tor }}=0.5$. Different colors indicate different ranges of $\tau=Z_{e f f} T_{e} / T_{i}$. c) Experimental, TEM [2] and ETG [29] $R / L_{T e}$ threshold as a function of $\tau$.

role of electron-scale instabilities in electron heat transport in these low power plasmas, more amenable than high performance scenarios to detailed transport physics studies due to the lack of strong magneto-hydro dynamic activity and ELMs (Edge Localized Modes). A precise experimental quantification of electron heat transport properties such as critical gradient for ETG onset and stiffness level on JET is an essential piece of information to validate both GK models and the most recent TGLF (SAT1) [24, 25, 26] quasi-linear model, to support their application to high power scenario predictions in present and future devices. A first experimental indication of the role of ETG modes in JET plasmas will be reported as well as strong confirmations from gyrokinetic simulations, including a first multi-scale simulation of a JET discharge.

Experimental observations: A variety of experimental observations pointing to a significant role of ETG modes in determining $q_{e}$ has been gathered in a set of JET C-wall dedicated electron heat transport experiments in L-mode plasmas with toroidal magnetic field $B_{T} \sim 3.35 T$, plasma current $I_{p} \sim 2 M A$, safety factor at the magnetic surface enclosing the $95 \%$ of the poloidal magnetic flux $q_{95} \sim 5$, electron density in the plasma center $n_{e, 0} \sim 2-3.5 \mathrm{u} 10^{19} \mathrm{~m}^{-3}$ and different levels of Neutral Beam Injection (NBI) and Ion Cyclotron Resonance Heating (ICRH). In these experiments, mid-radius $q_{e}$ scans at constant total power were carried out by using on- vs off-axis ICRH power in ${ }^{3} \mathrm{He}-\mathrm{D}$ plasmas with $n_{3} \mathrm{He} / n_{e} \sim 18 \%$ to achieve mode conversion and pure electron heating, as discussed in detail in Ref. [7]. These $q_{e}$ scans allow to determine the $R / L_{T e}$ threshold for the onset of turbulent electron heat transport and the electron stiffness $[3,4]$. In addition, $T_{e}$ modulation was also carried out, to complement the information $[27,28]$. The first striking observation (Fig. 1a) is a remarkable correlation at the analyzed radius $\rho_{\text {tor }}=\sqrt{\left(\Phi / \pi B_{T}\right) /\left(\Phi / \pi B_{T}\right)_{\max }}=0.5$ (where $\Phi$ is the toroidal magnetic flux) between $R / L_{T e}$ and the parameter $\tau=Z_{\text {eff }} \mathrm{u} T_{e} / T_{i}$ ( $T_{i}$ being the ion temperature and $Z_{\text {eff }}$ the effective plasma charge), which is a key term in the ETG threshold [29]. The $\tau$ dependence dominates over dependences on other parameters that also vary in the dataset, including the power level. The stabilizing effect of $\tau$ for electron heat transport can be seen in more detail in Fig. 1b, showing the gyro-Bohm normalized electron heat flux $q_{e, g B}=q_{e} /\left(n_{e} T_{e} c_{s} \rho_{s}^{2}\right)$, where $c_{s}=\sqrt{T_{e} / M_{i}}$ and $\rho_{s}=M_{i} c_{s} / e B, M_{i}$ being the ion mass, as a function of $R / L_{T e}$ and for different $\tau$ values. Here a clear increase of the electron threshold (i.e. the intercept at $q_{e, g B}=0$ ) is seen for increasing $\tau$, dominating over the scatter due to having included all points in the database, regardless of variations in other parameters (except $B_{T}$ and $I_{p}$ which are always the same). A consistent sound observation is that adding NBI power to an ICRH electron heated plasma induces a flattening of the $T_{e}$ profile and an increase of the electron stiffness, as reported in Ref. [7, 30]. Last, using a critical gradient formula [31] and the electron stiffness measured experimentally for few cases using $q_{e}$ scans and $T_{e}$ modulation, the $R / L_{T e}$ thresholds have been calculated for these discharges. These thresholds are compared, as a function of $\tau$, in Fig. 1c, with the theoretical values using analytical formulae proposed in Ref. [2] for TEM and in Ref. [29] for ETGs. We see that experimental thresholds lie close to the ETG threshold and have a similar trend with $\tau$ up to $\tau \sim 4$, whilst TEM thresholds have an opposite trend with $\tau$. 


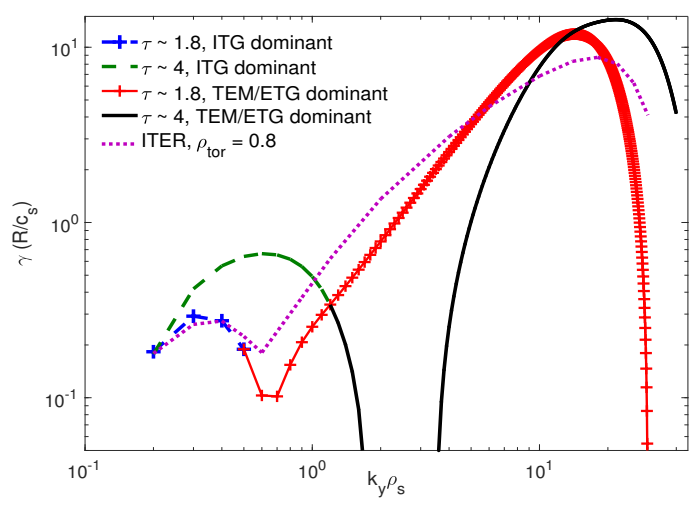

Figure 2: Normalized linear growth rate of the main instability from GENE linear simulations at $\rho_{\text {tor }}=0.5$ for $\tau=1.8$ (black) and $\tau=4.0$ (red). A simulation using ITER parameters from Ref. [33] at $\rho_{\text {tor }}=0.8$ is also shown, indicating that the linear growth rate of ion and electron-scale instabilities is similar to what found in JET. In the low $\tau$ JET case $L_{T i}^{I T G, c r i t} / L_{T i}=4.5 / 3$ and $L_{T e}^{E T G \text {,crit }} / L_{T e}=8.5 / 4.5$, while in the ITER case $L_{T i}^{I T G, \text { crit }} / L_{T i}=5.5 / 3.6$ and $L_{T e}^{E T G, \text { crit }} / L_{T e}=8.3 / 5.4 . R / L_{T i}^{I T G, \text { crit }}$ and $R / L_{T e}^{E T G, c r i t}$ indicate respectively the ITG and the ETG linear threshold calculated respectively at $k_{y} \rho_{s}=0.3$ and $k_{y} \rho_{s}=20$.

The experimental observations presented above are suggestive of a dominant role of ETG in electron heat transport in our dataset, but the quantitative evaluation of the relative impact of ITG, TEM and ETG modes on $q_{e}$ requires comparing the experimental plot of $q_{e, g B}\left(R / L_{T e}\right)$ (at constant other parameters) with non-linear gyrokinetic simulations.

Gyrokinetic simulation setup: For detailed non-linear simulations, carried out using the GENE code [6] in the radially local limit, input data from JET discharge n. 78834 (with 2.5 MW of ICRH power directed to electrons and with $1.7 \mathrm{MW}$ of NBI power) at $t=7 \mathrm{~s}$ and $\rho_{\text {tor }}=0.53$ have been used. Non-linear single-scale (on both ion and electron scales) and a first JET non-linear multi-scale simulation have been performed. A scan in $R / L_{T e}=8.5,10,11$ (experimental value: $R / L_{T e}=9.3 \pm 0.8$ ) has been done in the simulations. Other important parameters are $s=0.98, q=2.1, R / L_{n}=2.1, R / L_{T i}=3.6, T_{i} / T_{e}=0.88, Z_{\text {eff }}=1.82, \gamma_{E x B}=-r_{0} / q \mathrm{u} \partial \Omega / \partial r \mathrm{u} R / c_{s}=$ $0.01, \beta_{e}=8 \pi n_{e} T_{e} / B_{0}^{2} \approx 1 e-04$, where $s$ is the magnetic shear, $r_{0}$ is the minor radius of the considered flux surface and $\gamma_{E x B}$ is the $E \times B$ shear due to the plasma rotation $\Omega$. The low value of $\beta$ justify the electrostatic approximation used in the simulations. The simulations are electrostatic and feature Miller geometry, collisions, kinetic D ions and electrons and perpendicular flow shear rate. In order to cover both ion and electron scales, toroidal mode numbers up to electron-scale have been coupled in the multi-scale simulation, using $0.1 \lesssim k_{y} \rho_{s} \lesssim 48$. In the single ion and electron-scale simulations $0.05 \lesssim k_{y} \rho_{s} \lesssim 2.4$ and $3 \lesssim k_{y} \rho_{s} \lesssim 48$ have been used respectively. Perpendicular box sizes were $\left[L_{x}, L_{y}\right] \approx[64,64] \rho_{s}$ in the ion-scale and multi-scale simulations and $\left[L_{x}, L_{y}\right] \approx[4,4] \rho_{s}$ in the electron-scale simulations $\left(\rho_{s}=c_{s} / \Omega_{i}, c_{s}=\left(T_{e} / m_{i}\right)^{0.5}\right.$ and $\Omega_{i}=e B_{0} / m_{i}, m_{i}$ being the $\mathrm{D}$ mass and $B_{0}$ being the background magnetic field). The number of grid points used in the multi-scale simulation were $\left[n_{x}, n_{y}, n_{z}, n_{v}, n_{\mu}\right]=[1280,448,36,32,12]$ (GENE field-aligned coordinates: $x=$ radial direction, $y=$ binormal direction, $z=$ parallel direction (to $B_{0}$ ), $v=$ parallel velocity, $\mu=$ magnetic moments). In the single-scale simulations $\left[n_{x}, n_{y}, n_{z}, n_{v}, n_{\mu}\right]=[256,48,36,32,12]$. In the electron-scale simulations, the saturation of $q_{e}$ has been achieved by artificially increasing the level of the perpendicular flow shear until heat flux convergence was reached (using $\gamma_{E x B}=0.048$ ), due to ETG streamer shearing leading to a reduction of box-scale effects [32]. This technique has been assumed to be a proxy for ion-scale structures saturating the ETG streamers. The total predicted $q_{e, g B}$ from single-scale simulations has then been calculated with a simple sum of the fluxes from ion-scale and electron-scale simulations. Convergence tests have been made for the single-scale simulations but not for the multi-scale simulations due to the too high amount of computational resources that they would require.

Gyrokinetic simulation results: A first assessment of linear stability using GENE for plasmas at different values of $\tau$ is shown in Fig. 2. In the simulations, C has been used as an impurity species in the plasma 


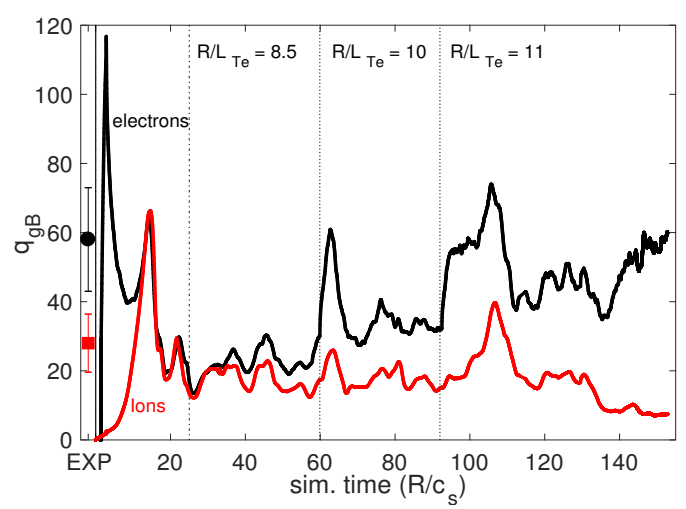

Figure 3: Multi-scale time evolution of $q_{i, g B}$ (red) and $q_{e, g B}$ (black). The three phases of the simulation with $R / L_{T e}=$ 8.5, 10,11 are indicated. The experimental $q_{i}$ and $q_{e}$ are shown on the left (EXP). The simulation required $\sim 20.000 .000$ CPU-hours.

in order to reproduce the experimental levels of $Z_{\text {eff }}$. In general, ITGs tend to be the dominant low-k mode for $k_{y} \lesssim 0.7$. For higher $k_{y}$ values TEM/ETG dominate. In many cases, ETGs have been found unstable also for high $\tau$ values, but a region with almost zero growth rate is present between the low and the high-k regions. For lower $\tau$ values, ITGs are in general more stable (in many cases due to an increase in $T_{i} / T_{e}[34]$ ), while TEM and ETG growth rates never decrease going from ion to electron scales. Considering that ETGs are expected to play a significant role when ion-scale instabilities are not highly unstable $[8,10,11]$, the results from the linear simulations go in the right direction to predict a stronger role of ETGs for lower $\tau$ values in these JET discharges. Regarding non-linear simulations, the time evolution of $q_{e, g B}, q_{i, g B}$ from the multi-scale simulation is shown in Fig. 3, while the comparison between non-linear simulations and experiment is shown in Fig. 6a. In both ion-scale and multi-scale simulations the $q_{i, g B}$ was matched within error-bars and no substantial differences in $q_{i, g B}$ have been observed when multi-scale interactions and high-k instabilities have been considered. The $q_{e, g B}$ predicted by ion-scale simulations is not enough to reproduce the experimental data. This was first pointed out in Ref. [7], but without any study of the electron-scale contribution, which is addressed in detail in this Letter. As shown in Fig. 6a, adding the $q_{e, g B}$ from the electron-scale simulations it is possible to reproduce both the $q_{e, g B}$ level and the electron stiffness. When multi-scale interactions are considered, no big differences in $q_{e, g B}$ with respect to the single ion-scale simulations have been observed for $R / L_{T e}=8.5$, while a strong increase of $q_{e, g B}$ has been observed with respect to the ion-scale simulations for $R / L_{T e}=10(+50 \%)$ and $R / L_{T e}=11(+80 \%)$. These increments are due to an increase of the high-k instabilities as can be seen from the $\phi-T_{\|}$(electrostatic potential-parallel temperature fluctuations) cross-phases, from the appearance in the snapshot of $\Phi\left(x / \rho_{s}, y / \rho_{s}\right)$ of the typical ETG streamer structures and from the appearance in the density fluctuation of a second peak around $5<k_{y} \rho_{s}<10$ when $R / L_{T e}$ is increased (Fig. $4,5)$.

A strong reduction of the fluxes and of the stiffness is observed with respect to the sum of the single-scale simulations, indicating that the saturation of ETGs due to ion-scale turbulent structures is a key factor and must be considered, as reported also in $[8,11,10]$. To gauge this interplay, using only the net free energy received by a mode from the nonlinear interaction with all other modes, i.e. $\mathcal{T}\left(k_{x}, k_{y}, z\right)$, see previous works $[35,36]$ or the equivalent definition in [37], we define the overall flux of free energy across the $k_{\perp}(z)$ scales as $\Pi\left(k_{\perp}, z\right)=\int_{\left|\mathbf{k}_{\perp}(z)\right| \geq k_{\perp}} \mathcal{T}\left(k_{x}, k_{y}, z\right) d k_{x} d k_{y}$. Here, we absorb the z-depended Jacobian into $\mathcal{T}$ for simplicity. In Fig. 4e, the plot of the flux of the free energy across the $k_{\perp}$ scales, for each value of $z$, is shown for the case $R / L_{T e}=10$. As can be seen, the overall flux is directed from large to small scales (positive flux values indicate a direct cascade, while negative flux values denote an inverse cascade) with the main contribution from modes that coincide with the ITG modes $\left(0.2<k_{y} \rho_{s}<0.4\right)$ and ETG modes $\left(5<k_{y} \rho_{s}<10\right)$ peaks for $q_{e, g B}$. To gauge solely the contribution of the ETG $k_{y}$ modes to the overall flux of free energy across $k_{\perp}(z)$, we employ the same flux definition but restrict ourselves to the desired $k_{y}$ 


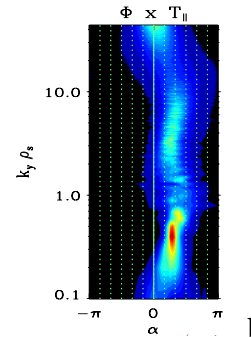

b)
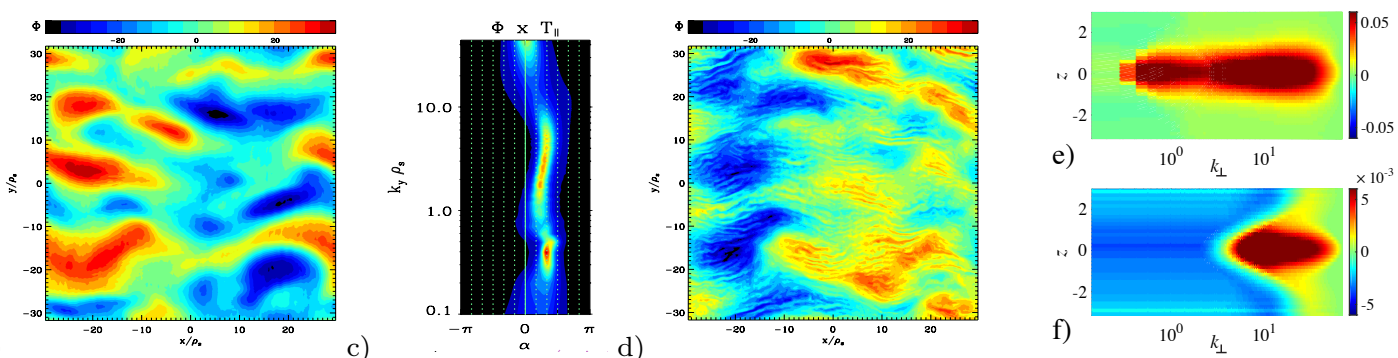

Figure 4: a, c) Cross-phases between $\Phi$ and $T_{e, \|}$ against phase angle $\alpha$ and $k_{y}$ for $R / L_{T e}=8.5(\mathrm{a})$ and $R / L_{T e}=11(\mathrm{c})$. The values are amplitude weighted. The red zones are contributing more to the flux. b, d) Snapshot of $\Phi\left(x / \rho_{s}, y / \rho_{s}\right)$ for $R / L_{T e}=8.5$ (b) and $R / L_{T e}=11$ (d). e)Free energy flux across perpendicular scales $k_{\perp}$ for each $z\left(\Pi\left(k_{\perp}, z\right)\right.$, normalized to $\left.\epsilon=\frac{1}{2} \int\left|\mathcal{T}\left(k_{x}, k_{y}, z\right)\right| d k_{x} d k_{y} d z\right)$ for $R / L_{T e}=10$ and (f) the contribution to $\Pi\left(k_{\perp}, z\right)$ from modes in the ETG range (i.e. contributions from modes with $5<k_{y} \rho_{s}<10$ in this case).

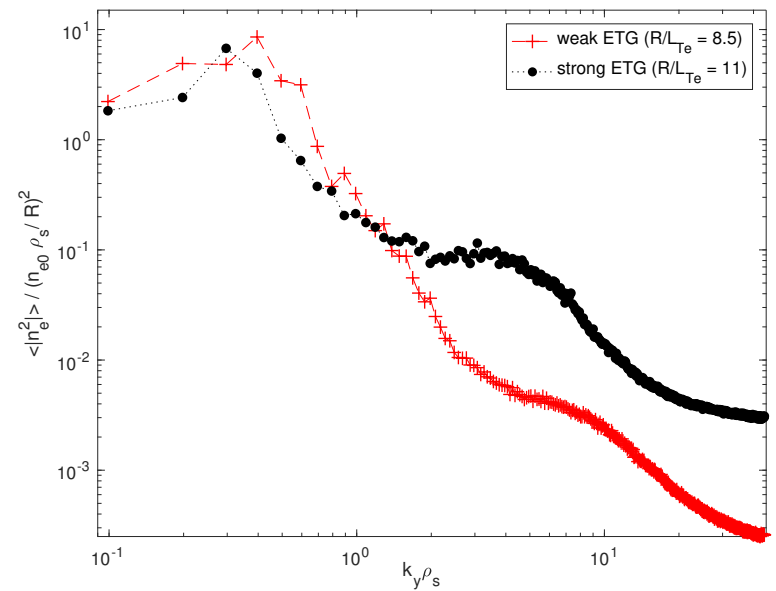

Figure 5: Density fluctuation $<\left|n_{e}^{2}\right|>_{x} /\left(n_{e, 0} \rho_{s} / R\right)^{2}$ at the outboard mid-plane $(z=0)$ as a function of $k_{y} \rho_{s}$ from the nonlinear multi-scale simulation for the cases $R / L_{T e}=8.5$ and $R / L_{T e}=11$. For $R / L_{T i}=11$, a second peak appears in the region $5<k_{y} \rho_{s}<10$ indicating a strong increase of the high-k instabilities.

range, i.e. $\mathcal{T}\left(k_{x}, k_{y} \in[5,10] / \rho_{s}, z\right)$. Looking at this ETG contribution (Fig. 4f), a $z$ dependent flux is visible. While a comprehensive analysis of scale interactions is desired, this simple diagnostic indicates that ETG modes can influence the ITG like scales through an inverse energy cascade process, which impacts the self-organization of structures (streamers) and the saturation level of $q_{e, g B}$, even when the overall scale flux is indicative of energy cascading down to smaller scales. This can be seen at $k_{\perp} \rho_{s} \sim 10$, where positive and negative flux values coexist for the same scale. These gyrokinetic results indicate that ETGs and multi-scale interactions can be important contributors to electron heat transport in these JET L-mode plasmas. The fact that quantitatively the $q_{e, g B}$ values of the multi-scale simulation are still lower than the experimental ones should not be seen as a failure of the multi-scale simulation, but rather as an indication that more ITG stabilizing mechanisms have to be introduced in the simulation, such as the presence of light impurities in the plasma $\left(C,{ }^{3} \mathrm{He}\right.$ in this case) $[38,39]$. These have not been included for sake of numerical resources. As indicated in Ref. [8, 10, 11], when ITG modes are close to threshold, a strong increment of both electron and ion heat flux can be observed. In our case, lower values of $R / L_{T i}$, outside error bars, would be needed in the present multi-scale simulation. However, adding impurities would lead to further stabilization of ITG modes whilst keeping the experimental $R / L_{T i}[38,39]$. This could lead to an enhancement of both electron and ion heat fluxes related to the presence of ETGs, with better agreement with experiment. This is left to future work.

Quasi-linear simulations: A study using the quasi-linear model TGLF has been done using the same input parameters and settings as in the GENE multi-scale simulation and the new TGLF saturation rule (sat 1) [26]. This new saturation rule takes into account the effect of ion-scale zonal flow on ETG instability and has been derived and 
tested using the multi-scale simulations reported in $[8,12]$. In the right plot of Fig. 6b, the comparison between TGLF and the multi-scale simulation is shown, while in the left plot the level of $q_{i, g B}$ is shown. TGLF predicts a strong impact of ion-scale ZF on ETGs, as can be seen by the large variation of $q_{e, g B}$ with $R / L_{T i}$ (different colors and symbols in the figure), with large ETG fluxes when ions are close to marginality. When $q_{i, g B}$ is matched between TGLF and the multi-scale simulation, the predictions for $q_{e, g B}$ are in good agreement (green diamonds and black pentagons). In this case a larger value of $R / L_{T i}$ ( 5 instead of 3.6 as in the gyrokinetic simulations) was needed in order to match the experimental flux. Furthermore, a strong increase of $q_{e, g B}$ due to ETGs is predicted by TGLF for higher values of $R / L_{T e}$, reaching the experimental level of $q_{e, g B}$ and also of the electron stiffness. The comparison of the electron heat flux between TGLF and the GENE ion-scale simulation (not shown) is also good. Both GENE and TGLF indicate that ion-scale instabilities alone are not explaining the experimental $q_{e, g B}$, and inclusion of electron-scales is essential.

Discussion: Experimental results in JET L-mode plasmas and their modelling with gyrokinetic simulations point to an important role of high-k instabilities and multi-scale interactions for electron heat transport. These findings represents a first proof of the role of ETGs in JET plasmas. Quasi-linear simulations are in good agreement, when the ion heat flux is matched, with the multi-scale GK simulation, indicating that these tools are useful for an at least qualitative prediction for ITER. Both the decrease of tau and the ITG stabilization due to increased rotation and non-linear e.m. stabilization [40], leading to enhanced ETG modes, may be the cause for the reduced value to which $T_{e}$ peaking is seen clamped in JET high NBI power performant scenarios. In JET, due to lower collisional coupling and to the presence of ion heating, this deterioration of electron heat transport is less penalizing for fusion performance than it could be in ITER. Indeed simple linear GK simulations of the ITER baseline plasma with the parameters predicted in [33] yield a ratio of high to low $\mathrm{k}$ growth rates comparable to what observed in these JET plasmas (Fig. 2), suggesting a similarly non-negligible role of ETGs. Therefore, these findings call for the need of properly accounting for high-k instabilities and multi-scale interactions in future ITER simulations and also of foreseeing ITER turbulence diagnostics covering reliably both low and high $\mathrm{k}$ ranges.

Acknowledgements: The authors would like to thank B. Baiocchi, G. Szepesi, E. Fable and G. Staebler for their help with the TGLF simulations and N. T. Howard for precious discussions. The authors are grateful to D. R. Mikkelsen for assistance. This research used resources of the NERSC center, a DOE Office of Science User Facility supported by the Office of Science of the U.S. Department of Energy under Contract No. DE--AC02--05CH11231. A part of this work was carried out using the HELIOS supercomputer system at IFERC-CSC, Aomori, Japan, under the Broader Approach collaboration between Euratom and Japan, implemented by Fusion for Energy and JAEA. We acknowledge the CINECA award under the ISCRA initiative, for the availability of high performance computing resources and support. B. Teaca is partially supported by EPSRC grant No. EP/P02064X/1. This work has been carried out within the framework of the EUROfusion Consortium and has received funding from the Euratom research and training programme 2014-2018 under grant agreement No. 633053. The views and opinions expressed herein do not necessarily reflect those of the European Commission. 


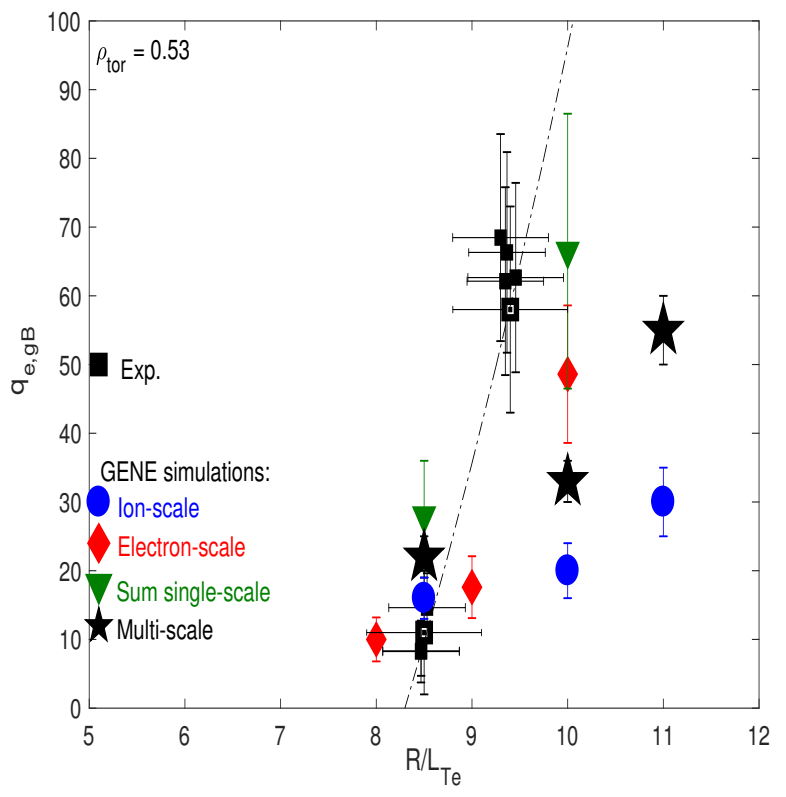

a)
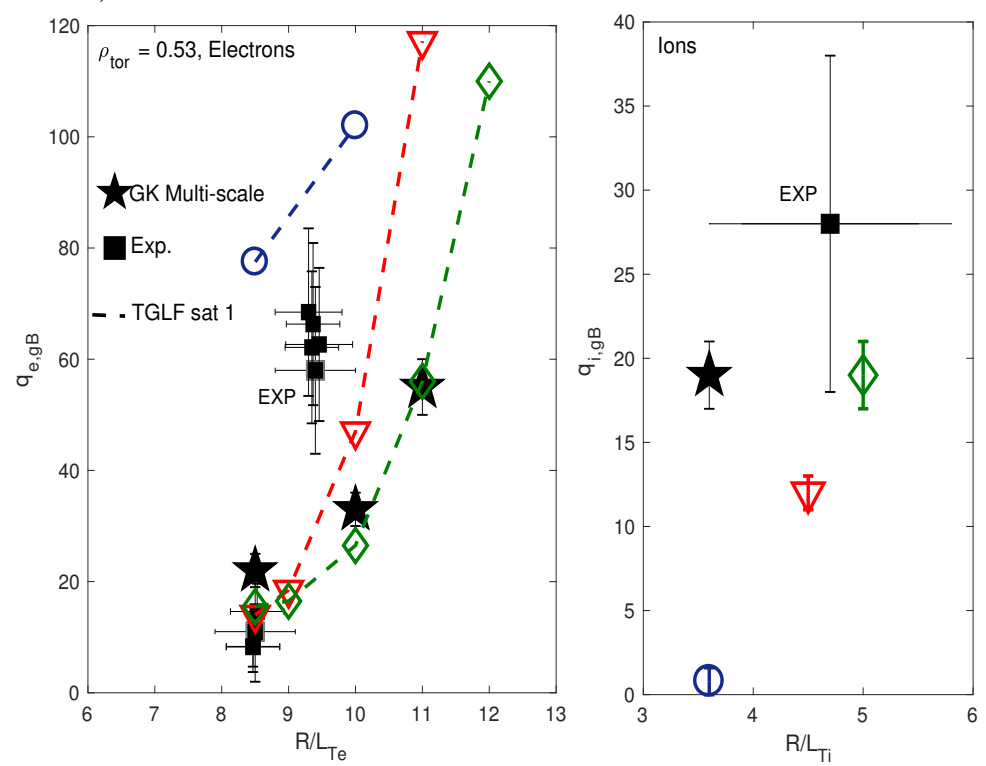

b)

Figure 6: a) $q_{e, g B}\left(R / L_{T e}\right)$. Comparison between experiment (black squares), gyrokinetic single-scale simulations (blue circles, green triangles and red diamonds) and gyrokinetic multi-scale simulations (black pentagons).

In the electron-scale simulations, a value of $\gamma_{E x B}=0.048$, four times the experimental ones, has been used in order to saturate the fluxes. b) Comparison of $q_{e, g B}\left(R / L_{T e}\right)$ (right) and $q_{i, g B s}\left(R / L_{T i}\right)$ (left), between gyrokinetic multi-scale simulations and TGLF-sat1 simulations. Using TGLF, scans in $R L_{T e}$ at different values of $R / L_{T i}$ have been performed (blue circles: $R / L_{T i}=$ 3.6 ; red triangles: $R / L_{T i}=4.5$; green diamonds: $\left.R / L_{T i}=5\right)$. 


\section{References}

[1] Romanelli F., Phys. Fluids B 1, 1018 (1989)

[2] Peeters A. G. et al., Phys. Plasmas 12, 022505 (2005)

[3] F. Ryter et al., Plasma Phys. Control Fusion 48, B453 (2006)

[4] P. Mantica et al., Fusion Science and Technology 53, 1152 (2008)

[5] J. C. Deboo et al., Phys. Plasmas 19, 082518 (2012)

[6] F. Jenko, et al., Phys. Plasmas 7, 1904 (2000)

[7] N. Bonanomi et al., Nucl. Fusion 55, 113016 (2015)

[8] N. T. Howard et al., Physics of Plasmas 21, 112510 (2014)

[9] Smith S. P. et al., Nucl. Fusion 55, 083011(2015)

[10] T. Görler and F. Jenko, Phys. Rev. Lett. 100, 185002 (2008)

[11] S. Maeyama and Y. Idomura, Phys. Rev. Lett. 114, 255002 (2015)

[12] N. T. Howard et al., Nucl. Fusion 56, 014004 (2016)

[13] T. L. Rhodes et al., Physics of Plasmas 14, 056117 (2007)

[14] W. Horton et al., Nucl. Fusion 45, 976 (2005)

[15] A. D. Gurchenko and E. Z. Gusakov, Plasma Phys. Control. Fusion 52, 124035 (2010)

[16] D. G. Applegate et al., Physics of Plasmas 11, 5085 (2004).

[17] C M Roach et al 2009 Plasma Phys. Control. Fusion 51124020.

[18] W. Guttenfelder and J. Candy, Physics of Plasmas 18, 022506 (2011).

[19] J. C. Hillesheim et al 2016 Plasma Phys. Control. Fusion 58014020.

[20] J. L. Burch et al. , Science Vol. 352, Issue 6290, aaf2939, 2016.

[21] M.O.Leubner, Z.Voros, Multi-scale Dynamical Processes in Space and Astrophysical Plasmas, Astrophysics and Space Science Proceedings Vol.33, Springer-Verlag Berlin Heidelberg, 2012.

[22] C. H. K. Chen et al., Phys. Rev. Lett. 110, 225002 (2013).

[23] C. Holland et al., Nucl. Fusion 57 (2017) 066043.

[24] G. M. Staebler et al., Phys. Plasmas 12, (2005)

[25] G. M. Staebler et al., Phys. Plasmas 14, (2007)

[26] G. M. Staebler et al., Phys. Plasmas 23, (2016)

[27] Mantica P. and Ryter F., C. R. Phys. 7, 634 (2006)

[28] Lopes N.J., Plasma Phys. Control. Fusion 37, 799 (1995) 
[29] F. Jenko, et al., Phys. Plasmas 8, 4096 (2001)

[30] X. Garbet et al., Plasma Phys. Control. Fusion 46, B557 (2006)

[31] Imbeaux F., Ryter F. and Garbet X., Plasma Phys. Control. Fusion 43, 1503 (2001)

[32] N. Bonanomi et al., EPS 2015, Lisbon, Portugal, 22-26 June 2015. Europhysics Conference Abstracts Vol. 39E, $\mathrm{P} 2-122$.

[33] B. Baiocchi et al., 2016 Joint Varenna-Lausanne International Workshop on the Theory of Fusion Plasmas, Varenna, Italy (Invited talk) and final report of ITER contract IO/CT/4300001087.

[34] S.C. Guo and F. Romanelli, Phys. Fluids B 5 (2), 520 (1993).

[35] Navarro A. B., Teaca B. and Jenko F., Phys Plasmas 23, 042301 (2016).

[36] Teaca B. et al., New J. Phys. 19, 045001 (2017).

[37] S. Maeyama et al., Nucl. Fusion 57, 066036 (2017).

[38] N. Bonanomi et al., Nucl. Fusion 58, 026028 (2018)

[39] Ennever P., et al., Phys. Plasmas 22, 072507 (2015).

[40] J. Citrin et al., Phys. Rev. Lett. 111, 155001 (2013) 